\title{
RELATÓRIO
}

\section{ARQUIVOS DE NEURO-PSIQUIATRIA 1999 - 2001}

\begin{abstract}
ARQUIVOS DE NEURO-PSIQUIATRIA (Arq Neuropsiquiatr, ISSN 0004-282X) é o periódico de ciência e tecnologia publicado trimestralmente pela Associação Arquivos de Neuro-Psiquiatria Dr. Oswaldo Lange, sociedade civil de fins não lucrativos, de que sou o Diretor Presidente. Ele é o periódico oficial da Academia Brasileira de Neurologia (ABN), a sociedade que em plano nacional reune os profissionais de saúde da área de Neurologia e que participa, pelo Brasil, da World Federation of Neurology (WFN), entidade internacional ligada à Organização Mundial de Saúde.

Dessa forma, o periódico se encontra credenciado à comunicação científico-tecnológica na área de conhecimentos em neurologia e neurociências: - em plano nacional, pela ABN; - em plano internacional, pela WFN.

Desse periódico tenho a honra de ser o Editor e é nesta qualidade que apresento este relatório. Abrange ele a produção técnico-científica havida em 1999 (Volume 57), 2000 (Volume 58) e 2001 (Volume 59).
\end{abstract}

\section{DESEM PENHO}

Publicação - Em 1999 (Vol.57), 2000 (Vol.58) e 2001 (Vol.59) foram publicados todos os números trimestrais de Arquivos de Neuro-Psiquiatria: M arço (N. 1), J unho (Ns. 2-A e 2-B), Setembro (Ns. 3-A e 3-B) e Dezembro (N. 4). Os números de junho e de setembro têm as duas partes mencionadas (fascículos A eB), com o que se buscou atender ao aumento crescente de artigos submetidos e aceitos para publicação após revisão dentro do sistema de arbitragem por pares.

Tiragem - A tiragem anual exemplares / número foi: 1800 em 1999, 1900 em 2000 e 1950 em 2001.

Regularidade, periodicidade, normas técnicas - Todos os números foram publicados nos respectivos meses de capa, mantendo-se assim a regularidade e periodicidade absolutas do periódico desde sua criação (1943) até o presente. Ademais, foram mantidas as normas técnicas de publicação preconizadas pelo CNPq, as quais obedecem às recomendações internacionais para tanto $e$, no que cabe, àquelas do IBICT e da ABNT.

Linha editorial - Dentro da linha editorial do periódico, todos os artigos publicados são inéditos eforam previamente avaliados dentro do sistema de arbitragem por pares.

Objetivos e propósitos - Dentro dos objetivos e propósitos do periódico, os números prioritariamente contêm artigos originais de natureza científico-tecnológica e de interesse à neurologia e neurociências aplicadas.

Matéria publicada - A matéria publicada se distribui em duas seções; a seção de artigos originais, prioritária; a seção geral. O conteúdo dos dois grupos consta dos itens seguintes.

Artigos - Em 1999 (Vol.57) apareceram 169 artigos originais: 26 no N.1, 29 no N.2-A, 30 no N.2-B, 28 no N. 3-A, 30 no N. 3-B e 26 no N.4. Em 2000 (Vol. 58), apareceram 179 artigos originais: 30 no N.1, 29 no N.2-A, 30 no N.2-B, 31 no N.3-A, 30 no N.3-B e 29 no N.4. Em 2001 apareceram 186 artigos originais: 30 no N.1, 32 no N.2A, 31 no N.2-B, 32 no N. 3-A, 35 no N. 3-B e 26 no N..4.

Seção geral - Os volumes incluem as seções especiais que seguem.

- Theses, - Em que foram publicados os abstracts de 21 teses em 1999 (Vol.57), 17 em 2000 (Vol.58) e 23 em 2002 (Vol.59).

- Correspondência - Em que foram publicadas cartas sobre artigos publicados e as respectivas respostas dos autores desses artigos: três em 1999 (Vol.57), duas em 2000 (Vol.58) e três em 2001 (Vol.59).

- Editoriais - Em que foram publicados: em 1999 (Vol.57) um, sobre perspectivas de pesquisa em uma das áreas de neurologia e um de Homenagem; em 2000 (Vol.58) um, de homenagem póstuma (In M emoriam ); em 2001 (Vol.59) um, de homenagem póstuma (In M emoriam).

- Análises de Livros - Nesta seção aparecem, em cada número, avaliações de livros de interesse para a área, tendo sido analisados: 30 compêndios em 1999 (Vol.57); 21, em 2000 (Vol.58); 23, em 2001 (Vol.59).

- Notícias e Comentários - Em que aparecem dados de interesse para a área, publicada nos fascículos de 1999 (Vol. 57), 2000 (Vol. 58) e 2001 (Vol. 59).

- Agradecimento aos Avaliadores - Esse agradecimento é publicado anualmente, no número Dezembro, juntamente com a lista dos que contribuíram durante o ano para a revisão do conteúdo científico e tecnológico dos artigos submetidos para publicação, dentro do sistema de arbitragem por pares.

- Índice Geral do Volume - Contendo índice de autores, índice de assuntos e subject index o Índice Geral do Volume, esta seção também aparece anualmente, encerrando o N.4 (Dezembro).

Distribuição da matéria - A distribuição da matéria pelo 
total de páginas de texto em cada um dos três volumes foi como segue.

- O total de páginas de texto do Vol.57 (1999) é 1076, assim distribuídas: 54 (5,0\%) para as seções de Análises de Livros e de Notícias e Comentários; $4(0,4 \%)$ para aquela de Correspondência, $8(0,8 \%)$ para a de Editoriais, 16 (1,5\%) para a de Theses; e 996 (92,3\%) os 169 artigos.

- O total de páginas de texto do Vol.58 (2000) é 1176, assim distribuídas: 40 (3,4\%) para as seções de Análises de Livros e de Notícias e Comentários; 3 (0.3\%) para aquela de Correspondência, $7(0,6 \%)$ para a de Editoriais, 16 (1,4\%) para a de Teses; e 1110 (94,3\%) os 179 artigos.

- O total de páginas de texto do Vol.59 (2001) é 1008, assim distribuídas: 38 (3,8\%) para as seções de Análises de Livros e de Notícias e Comentários; 3 (0,3\%) para aquela de Correspondência, $7(0,7 \%)$ para a de Editoriais, 19 (1,9\%) para a de Teses; $18(1,8 \%)$, para o Índice; e 923 (91,5\%) para os 186 artigos.

Título, Resumo, Descritores - Os artigos em português ou espanhol têm título alternativo em inglês e aqueles em inglês, em português ou espanhol. Todos os artigos têm key words e palavras-chave ou palabras-clave.

Idioma - Os artigos aparecem em português, em inglês ou em espanhol. Em 1999 (Vol.57) os 169 artigos aparecem em português (110) ou em inglês (59). Em 2000 (Vol.58), os 179 artigos apareceram em português (102), em inglês (76) ou em espanhol (1). Em 2001 (Vol.59), os 186 artigos aparecem em português (96), em inglês (89) ou em espanhol (1).

\section{ARTIGOS: CONTEÚDO E PROCEDÊNCIA}

Em 1999 (Vol.57), todos os 169 artigos são inéditos: $160(94,7 \%)$ registram os resultados originais de pesquisas clínicas ou experimentais; 9 (5,3\%) são artigos de opinião e crítica, baseados no conhecimento de seus autores acerca dos temas abordados. Todos os artigos foram aceitos para publicação após serem avaliados por revisores especializados na matéria específica de que tratam (revisão por pares): 308 revisores gentilmente avaliaram, duranteo ano, artigos submetidos para publicação; seus nomes constam do Agradecimento aos Avaliadores publicado no N.4 (Dezembro), pgs 1065-1068. Seus esforços beneficiaram direta ou indiretamente tanto os 169 artigos como seus autores.

Os 169 artigos publicados resultaram de estudos desenvolvidos em centros de pesquisa do Brasil (165), ou da colaboração entre centros estrangeiros e brasileiros (3) e em centros estrangeiros (1). Os 165 estudos desenvolvidos em centros de pesquisa brasileiros provêm de instituições situadas no Distrito Federal (2) e nos Estados de: São Paulo (67), Paraná (27), Rio de Janeiro (20), Minas Gerais (13), Santa Catarina (7), Rio Grande do Sul (7), Bahia (5), Ceará (5), Goiás (2), Mato Grosso (2), Paraíba (2), Mato Grosso do Sul (1), Pernambuco (1), São Paulo e Mato Grosso do Sul (2), São Paulo e Pará (1), Rio Grande do Sul, Rio de Janeiro e São Paulo (1). Os três artigos resultantes de estudos de colaboração internacional de centros de pesquisa provêm da conjugação de esforços de centros universitários de pesquisa dos EUA e do Estados do Paraná (1) e Rio de Janeiro (1), da Austrália e do Estado de São Paulo (1). O artigo de centro de pesquisa de outro país foi desenvolvido na Austrália.

Em 2000 (Vol.58) todos os 179 artigos são inéditos: $173(96,6 \%)$ registram os resultados originais de pesquisas clínicas ou experimentais; 6 (3,4\%) são artigos de opinião e crítica ou de consenso, baseados no conhecimento de seus autores acerca dos temas abordados. Todos os artigos foram aceitos para publicação após serem avaliados por revisores especializados na matéria específica de que tratam (arbitragem por pares): 362 revisores gentilmente avaliaram, durante $o$ ano, artigos submetidos para publicação; seus nomes constam do Agradecimento aos Avaliadores publicado no N.4 (Dezembro), pgs 1169-1173. Seus esforços beneficiaram direta ou indiretamente tanto os 179 artigos como seus autores.

Os 179 artigos publicados provieram de centros de pesquisa do Brasil (171) ou do exterior (8). Os 171 estudos provenientes de centros de pesquisa brasileiros se originaram no Distrito Federal (2) e nos Estados de: São Paulo (81), Paraná (30), Rio de Janeiro (22), Minas Gerais (13), Rio Grande do Sul (7), Bahia (3), Ceará (3), Espirito Santo (2), Santa Catarina (2), Maranhão (1), Mato Grosso (1), Mato Grosso do Sul (1), Paraíba (1), Pernambuco (1), Sergipe (1). Os 8 artigos provenientes de centros de pesquisa do exterior se originaram nos países: Argentina (2), Austrália (1), Cuba (1), Estados Unidos (3), França (1).

Em 2001 (Vol.59) todos os 186 artigos são inéditos: $180(96,8 \%)$ registram os resultados originais de pesquisas clínicas ou experimentais; 6 (3,2\%) são artigos de opinião e crítica ou de consenso, baseados no conhecimento de seus autores acerca dos temas abordados. Todos os artigos foram aceitos para publicação após serem avaliados por revisores especializados na matéria específica de que tratam (arbitragem por pares): 381 revisores gentilmente avaliaram, durante o ano, artigos submetidos para publicação; seus nomes constam do Agradecimento aos Avaliadores publicado no N.4 (Dezembro), pgs 987-991. Seus esforços beneficiaram direta ou indiretamente tanto os 186 artigos como seus autores.

Os 186 artigos publicados provieram de centros de pesquisa do Brasil (179) ou do exterior (7). Os 179 estudos provenientes de centros de pesquisa brasileiros se originaram nos Estados de: São Paulo (76), Paraná (44), Rio de Janeiro (22), Minas Gerais (10), Rio Grande do Sul (9), Pernambuco (5), Bahia (4), Paraíba (3), Ceará (2), Santa Catarina (2), Amazonas (1), Mato Grosso do Sul (1). Os 6 artigos provenientes de centros de pesquisa do exterior tiveram com país de origem: Estados Unidos (3), Argentina (1), Austrália (1), Colômbia (1), Uruguai (1).

ABSTRACTS DE TESES:

NATUREZA E PROCEDÊNCIA

Em 1999 (Vol.57), as teses cujos 21 abstracts foram publicados, foram aprovadas em instituições universitári- 
as do Brasil (20) e de Portugal (1). Doze eram abstracts de Dissertações de Mestrado dos cursos de pós-graduação da: Faculdade de Medicina de São José do Rio Preto (2); Universidade de Brasília (1); Universidade de Federal de São Paulo, UNIFESP (1); Universidade Federal do Paraná, UFPR (1); Universidade Federal Fluminense, UFF (1); Universidade Federal de Minas Gerais, UFMG (1) Universidade de São Paulo (USP), nas Faculdades de Medicina de Ribeirão Preto, FMRPUSP (1) e de São Paulo, FMUSP (1), Universidade Estadual de São Paulo, UNESP (1), Pontifícia Universidade Católica do Rio Grande do Sul, PUCRS (1); do Instituto de Assistência Médica do do Estado de São Paulo, IAMSPE (1). Oito eram abstracts Teses de Doutorado dos cursos de pós-graduação da: FMUSP (4); UFF (1); UNESP (1); Universidade Estadual de Campinas, UNICAMP (1); Universidade do Porto, Portugal (1). Um era abstract de Tese de Docência Livre em Neurologia da UNESP.

Em 2000 (Vol.58), as 17 teses cujos abstracts foram publicados, foram aprovadas em instituições universitárias do Brasil. Nove são abstracts de Dissertações de Mestrado dos cursos de pós-graduação da: Universidade Federal Fluminense, UFF (2), Universidade Federal do Rio de Janeiro, (2), Universidade de São Paulo, USP (2), Universidade Federal do Rio Grande do Sul (1), Universidade de Brasília (1), Universidade de Marília (1). Cinco são abstracts Teses de Doutorado dos cursos de pós-graduação da: USP (3) e da UFF (2). Três são abstracts de Teses de Livre-Docência em Neurologia, da USP (2) e da Universidade Estadual de Campinas, UNICAMP (1).

Em 2001 (Vol.59), as 23 teses cujos abstracts foram publicados, foram aprovadas em instituições universitárias do Brasil. Onze são abstracts de Dissertações de Mestrado dos cursos de pós-graduação da: Universidade Federal Fluminense, UFF (2), Universidade de São Paulo, USP (2), Faculdade de Medicina de São J osé do Rio Preto, FAM ERP (1), Santa Casa de Belo Horizonte (1), Universidade de Brasília (1), Universidade Estadual de São Paulo, UNICAMP (1), Universidade Federal de Pernambuco, UFPE (1), Universidade Federal do Rio de Janeiro, UFRJ (1), Universidade Federal do Rio Grande do Sul, UFRS (1). Nove são abstracts de Teses de Doutorado dos cursos de pósgraduação da USP (3), UFR (2), Universidade Federal de São Paulo, UNIFESP (2) FAMERP (1), UFRS (1). Dois são abstracts de Teses de Livre-Docência em Neurologia, da USP. Um é abstract de Tese de Professor Titular da UFPE.

\section{AGÊNCIAS FINANCIADORAS DE PESQUISA}

Em 1999 (Vol.57), em 15 artigos é reconhecido o auxílio à pesquisa ou ao pesquisador recebido de agências oficiais de financiamento e de amparo à pesquisa em ciência e tecnologia do Brasil: CNPq (9), FAPEMIG (3) FAPESP (2), CAPES (1).

Em 2000 (Vol.58), em 29 ocasiões é reconhecido em artigos o auxílio à pesquisa ou ao pesquisador recebido de agências oficiais de financiamento e de amparo à pesquisa: CNPq (8), CAPES (3), FAPESP (9), outras agências do Brasil ou do exterior (9).

Em 2001 (Vol.59), em 27 ocasiões é reconhecido em artigos o auxílio à pesquisa ou ao pesquisador recebido de agências oficiais de financiamento e de amparo à pesquisa: CNPq (8), CAPES (3), FAPESP (10), outras agências do Brasil ou do exterior (6).

\section{DISTRIBUIÇÃO E CIRCULAÇÃO}

A distribuição dos 1800 exemplares / número de 1999 incluiu: 221 bibliotecas (nacionais 147, sendo 59 assinantes; estrangeiras 74, sendo 22 assinantes); 58 permutas com outros periódicos (nacionais, 11; estrangeiros, 47); 1426 assinantes. Os 95 exemplares restantes serviram para divulgação (45) ou reserva operacional (50).

A distribuição dos 1900 exemplares / número de 2000 incluiu: 249 bibliotecas (nacionais 172, sendo 95 assinantes; estrangeiras 77, sendo 22 assinantes); 73 permutas com outros periódicos (nacionais, 25; estrangeiros, 48); 1498 assinantes. Os 80 exemplares restantes serviram para divulgação (30) ou reserva operacional (50).

A distribuição dos 1950 exemplares/ número de 2001 atingiu: 247 bibliotecas (nacionais 147, sendo 83 assinantes; estrangeiras 100, sendo 38 assinantes), 73 permutas com outros periódicos (nacionais, 25 ; estrangeiros, 48), 1553 assinantes. Os 77 exemplares restantes serviram para divulgação (37) ou reserva operacional (40).

\section{DEMANDA DE PUBLICAÇÃO}

Em 1999 foram submetidos 209 artigos; 163 foram publicados (67 durante o ano e os restantes, posteriormente); 33 foram recusados; 13 não foram reencaminhados por seus autores após os pareceres dos revisores e dos editores.

Em 2000 foram submetidos 274 novos artigos: 201 foram publicados (86 publicados durante o ano; 115, posteriormente); 46 foram recusados; 27 não foram reencaminhados por seus autores após os pareceres dos revisores e editores.

Em 2001 foram submetidos 257 novos artigos: 193 foram publicados (66 durante 2001; 167 em 2002); 48 foram recusados; 16 não foram reencaminhados por seus autores após os pareceres dos revisores e dos editores.

Este é o relatório. 